\title{
Reversal of Alcoholic Fatty Liver in Patients with Acute Pancreatitis - A Prospective Observational Study
}

\section{Venkatesh Kasi Arunachalam*, Thambidurai Sadasivam, Rupa Renganathan, Gopinath Periaswamy, Haleema Sherene, Rajesh S, Rajesh Kumar Varatharajaperumal and Sriman Rajasekaran}

Department of Radiology, Kovai Medical Center and Hospital, Coimbatore, India

*Corresponding Author: Venkatesh Kasi Arunachalam, Senior Consultant Radiologist, Department of Radiology, Kovai Medical Center and Hospital, Coimbatore, India.
Received: June 17, 2021

Published: July 13, 2021

(C) All rights are reserved by Venkatesh Kasi Arunachalam., et al.

\section{Abstract}

Purpose of the Study: Fatty liver is a reversible condition characterized by the accumulation of triglycerides within the hepatocytes. The prime way to reverse the fatty liver includes abstinence from alcohol, dietary modifications, and regular exercise. Our study aimed to assess the reversal of alcoholic fatty liver in patients with acute pancreatitis.

Materials and Methods: One hundred five patients with acute pancreatitis were included in our study. The liver attenuation index was calculated in a plain scan, and fatty infiltration was quantified. All the patients with fatty infiltration were divided into an alcoholic and non-alcoholic subgroup. The LAI was calculated in the follow-up CT scan in these patients with fatty infiltration, and reversal of fatty liver was calculated along with the time.

Results: Among 105 patients with acute pancreatitis, 32 had fatty liver. In these 32 patients, alcohol was the cause of acute pancreatitis in 18 patients. Follow up CT scan was done in $40 \%$ of the patients with fatty infiltration. Out of 10 patients with fatty infiltration in an alcoholic subgroup, eight patients showed reversal of fatty liver in 4 days to 16 weeks, and all these patients were abstinent from alcohol from the time of admission.

Conclusion: Abstinence from alcohol is the mainstay in the reversal of alcoholic fatty liver, and it will take at least four weeks for the complete reversal of the alcoholic fatty liver disease.

Keywords: Alcoholic Fatty Liver; Reversal of Fatty Liver; Acute Pancreatitis; Liver Attenuation Index; Abstinence

\section{Abbreviations}

AFLD: Alcohol-Related Fatty Liver Disease; AH: Alcohol-Related Hepatitis; ALC: Alcohol-Related Cirrhosis; ALD: Alcoholic Liver Disease; LAI: Liver Attenuation Index; ALP: Alcohol-Related Pancreatitis; AAP: Acute Alcohol-Related Pancreatitis; ACP: AlcoholRelated Chronic Pancreatitis

\section{Introduction}

Fatty liver is a condition characterized by the accumulation of triglycerides within the hepatocytes. Though many conditions al- ter the hepatocellular lipid metabolism resulting in fat accumulation in the liver, the two most common causes of hepatosteatosis include alcoholic liver disease and Nonalcoholic fatty liver disease [1]. The fatty liver may progress to steatohepatitis and then to cirrhosis.

The most prevalent ways to stop the progression and reverse the fatty liver include abstinence from alcohol, dietary modifications, and regular exercise. The fatty liver is known to occur in $15 \%$ of the general population and $45 \%$ of the heavy drinkers [1]. The two 
most commonly injured digestive organs secondary to excessive alcohol consumption are the liver and pancreas [2,3]. Both these organs share several structural and functional similarities. The manifestations in the liver result in a spectrum of disease, which includes alcohol-related fatty liver disease (AFLD), alcohol-related hepatitis (AH), and alcohol-related cirrhosis (ALC) [4].

Similarly, the manifestation in the pancreas secondary to alcohol consumption includes alcohol-related acute and chronic pancreatitis. The prevalence of alcoholic liver disease (ALD) in Alcoholic pancreatitis corresponds to $39.0 \%$ [4]. The consequences of alcohol toxicity in both these organs are mainly secondary to acetaldehyde adducts, oxidative stress, and increased lipopolysaccharides, which results in parenchymal damage, activation of resident cells, and finally fibrosis $[5,6]$. As mentioned earlier, abstinence from alcohol is one of the primary treatment methods in patients with alcoholic liver disease (ALD). In patients with simple alcoholic fatty liver, abstinence will allow the liver to heal and return to normal. In alcohol-induced fibrosis or cirrhosis, abstinence from alcohol will decrease the damage to the liver. As a result, the liver functions will get better and will not degrade further. However, there is no significant change in the scar tissue in the liver parenchyma [7].

Though the advantages of abstinence from alcohol start immediately, it will take weeks to months to achieve in the complete sense in alcoholic liver disease, and the duration taken varies from patient to patient [7]. This prospective observational study aims to determine the percentage and duration taken to reverse alcoholic fatty liver in patients with acute alcoholic pancreatitis.

\section{Materials and Methods}

The local ethical and scientific committee approved this prospective observational study. All the patients referred to the radiology department with a clinical diagnosis of acute pancreatitis from Jan 2020 to May 2021 were included in this study. Informed consent was obtained from these patients. The patients with a previous history of pancreatitis were excluded from this study. The details such as age, sex, history of alcohol consumption, and abstinence from alcohol were collected. All these patients underwent dual-phase CT scans in third-generation dual-source dual-energy CT scan, which includes plain scan extending from dome of diaphragm to iliac crest and post-contrast scan in venous phase extending from diaphragmatic dome to pubic symphysis. Both these phases were acquired using single energy mode. The plain non-contrast images in these patients were evaluated for fatty liver by analyzing liver attenuation index (LAI) [8]. The patients were classified into three groups based on LAI; No fatty infiltration, mild to moderate fatty infiltration, and moderate to severe fatty infiltration. Few of these patients with fatty infiltration underwent follow-up CT scans, and they form the prime subgroup of patients in our study. The LAI was calculated in these scans and compared with the index scan.

\section{Results}

One hundred five patients were included in this study. In our study, $87 \%$ of patients were males (91 patients). The age group was from 25 - 74 years. In the 105 patients with acute pancreatitis, alcohol was the etiology in 33\% of patients. 32 out of 105 patients were diagnosed to have fatty liver. In 35 patients with a history of alcohol consumption, 18 patients had fatty liver, 2 had alcoholic cirrhosis and one had alcoholic hepatitis. In our series of patients with a history of alcohol consumption, 17 patients had normal attenuation, and 18 patients showed moderate to severe fatty infiltration. There was no patient with mild to moderate fatty infiltration in the alcoholic subgroup. In 14 patients with fatty liver in the nonalcoholic group, two patients showed mild to moderate fatty infiltration, and 12 showed moderate to severe fatty infiltration. Out of 32 patients with fatty infiltration, $40 \%$ of patients (13 patients) had follow-up CT scans. Of these 13 patients, ten were from the alcoholic group and three from the nonalcoholic group. The time between the index and follow-up CT scan ranges between 4 days to 16 weeks. There was no reversal of fatty liver in the nonalcoholic group, and the scans were done from 1 week to 16 weeks. Out of the ten patients from the alcoholic group with follow-up CT scan, reversal of steatosis was noted in 8 patients. In the follow-up CT scan done in less than ten days (in 2 patients), reversal of moderate to severe fatty infiltration to mild to moderate fatty infiltration was noted (Figure 1). In the rest of 6 patients, reversal of moderate to severe fatty infiltration to normal attenuation was noted (Figure 2). In these patients' follow-up, a CT scan was done between 6 - 16 weeks. 2 patients in the alcoholic group did not show reversal of fatty infiltration (Figure 3). When we correlated with history, all the eight patients in whom reversal of fatty liver demonstrated was abstinent from alcohol from the day of admission, and the two patients who did not show steatosis reversal restarted their alcohol consumption sometimes after their discharge. 


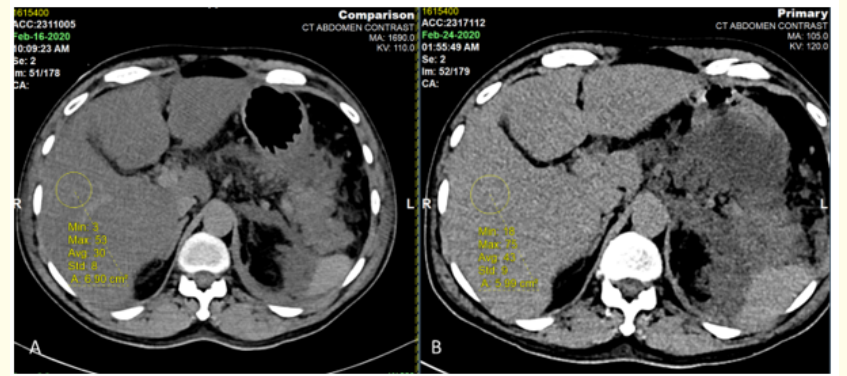

Figure 1: Axial non contrast CT images $(A \& B)$ in a patient with partial reversal of fatty liver: Region of interest (ROI) in the scan done on 24/02/2020 (B) show mild increase in attenuation of liver compared to CT scan done on 16/02/2020(A).

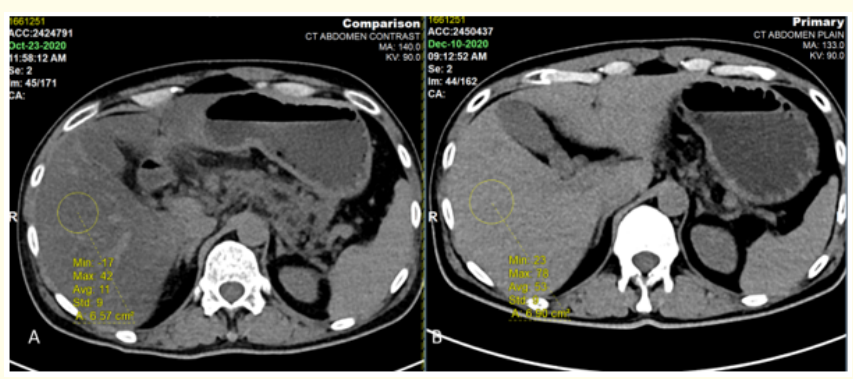

Figure 2: Axial non contrast CT images (A\&B) in a patient with complete reversal of fatty liver: Region of interest (ROI) in the scan done on 10/12/2020 (B) shows show significant increase in attenuation of liver compared to CT scan done on 23/10/2020(A).

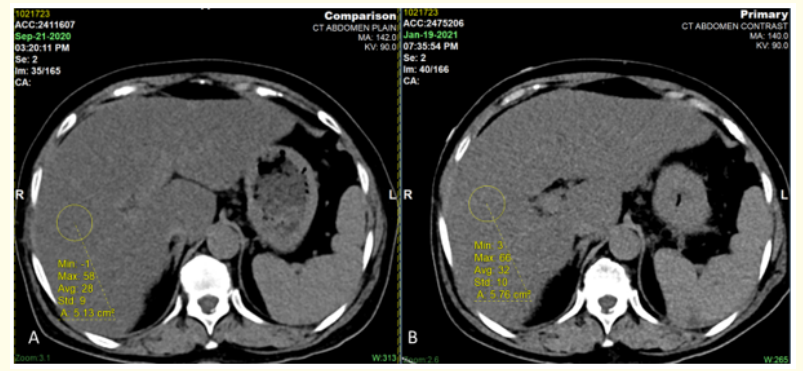

Figure 3: Axial non contrast CT images (A\&B) in a patient with no reversal of fatty liver: Region of interests (ROI) in the scans done on 23/10/2020(A) and 19/01/2021 (B) show no significant interval change in liver attenuation.

\section{Discussion}

Fatty liver or hepatic steatosis is a reversible condition in which triglycerides are accumulated within the cytoplasm of hepatocytes. The disorders of hepatic lipid metabolism, mainly free fatty acid metabolism, result in triglyceride accumulation within the hepatocytes. Fatty liver can be secondary to many causes, of which alcoholic fatty liver and nonalcoholic fatty liver disease are the two most essential etiologies. The fatty liver, if untreated, may progress to steatohepatitis and then to cirrhosis. The incidence of fatty liver in the general population is about $15 \%$. However, it is increased to $45 \%$ in heavy drinkers, $75 \%$ in obese individuals, and $90 \%$ in obese and heavy drinkers [9-15].

Alcohol abuse not only damages the liver but also many organs and presents a variety of phenotypes. It is a contributory factor in around 200 disease states [16]. The two most commonly injured organs by excessive alcohol consumption in the digestive system are the liver and pancreas $[17,18]$. Based on the pathologic findings, alcoholic liver disease is classified as fatty liver, alcoholic hepatitis, and alcoholic cirrhosis [19]. Similarly, Alcohol-related pancreatitis (ALP) is classified into acute alcohol-related (AAP) and alcohol-related chronic pancreatitis (ACP). One of the main reasons for liver damage is the concentrations of alcohol found in the portal blood. The concentration is more than those in the systemic circulation [20]. Since the liver is the primary site of alcohol metabolism, which results in many toxic metabolites, the liver is damaged primarily by alcohol toxicity.

Along with this direct injury, low-grade hepatic inflammation is also associated with ALD. This inflammation is incompletely compensated by regeneration [21]. In a meta-analysis study by Singhvi., et al. the pooled prevalence of ALD in ALP corresponds to $39.0 \%$. In our study, the prevalence of ALD corresponds to $20 \%$ of patients [4].

Fatty liver or steatosis is known to occur in about 45 percent of heavy drinkers [22]. In our series, 52\% of patients with an alcohol history had fatty liver. Patients with AFLD are usually asymptomatic except for hepatomegaly. Both hepatic and extrahepatic cause contributes to the development of fatty liver in alcoholics. The cause includes enhanced lipogenesis, impaired VLDL secretion, decreased fatty acid oxidation, reduced adipokine production, and increased fatty acid influx from adipose tissue [23]. 
The fatty liver can be diagnosed and graded using USG, CT, and MRI. We used the liver attenuation index in our study to grade the fatty liver. The liver attenuation index is the difference between the mean attenuation of the liver and the mean attenuation of the spleen.

If LAI > 5HU, it suggests the absence of significant macrovesicular steatosis. LAI between 5 to $-10 \mathrm{HU}$ suggests mild to moderate fatty infiltration (6-30\%), and <-10HU suggests moderate to severe fatty infiltration (> 30\%) [24]. In our study, 51\% of patients with alcoholic history had fatty liver, and they had moderate to severe fatty infiltration.

Fatty liver is a reversible disease. The mainstay of treatment in fatty liver disease includes abstinence from alcohol, decreasing calorie intake, and increased energy expenditure. In AFLD, abstinence leads to complete resolution of the fatty liver, while in alcoholic cirrhosis, it improves the survival of patients, even in those with decompensated liver disease. In AFLD, the reversal of steatosis usually occurs within 2-6 weeks of abstinence from alcohol [2527]. Nevertheless, the liver healing period may vary from patient to patient, and it may take weeks to months to achieve in the complete sense.

In our series, we calculated the LAI in the follow-up CT scans and compared it with the index scans. Out of 13 patients with follow-up CT, ten patients were from the alcoholic group. In these ten patients with fatty liver, $80 \%$ of patients showed reversal of fatty liver. The follow-up scan period ranged between 4 days to 16 weeks. In the patients with follow-up CT scan done less than two weeks, partial reversal was demonstrated. In the rest six patients, followup $\mathrm{Ct}$ was done between 4 weeks to 16 weeks and showed complete reversal. All these eight patients gave a history of abstinence from alcohol from the time of admission.

\section{Limitation of the Study}

Our study has few limitations. First, the sample size is small. Large-scale studies are needed to confirm the results. Second, we calculated the LAI in the follow-up scans done for some other reasons. Hence the follow-up scan period was varied between 4 days to 16 weeks. We need to do the follow-up scans for all the patients at a particular time to demonstrate the reversal of steatosis with the time.

\section{Conclusion}

Alcoholic fatty liver is a reversible disease. Abstinence from alcohol at least for four weeks results in the complete reversal of fatty liver. In early periods of abstinence of fewer than two weeks, a partial reversal can be demonstrated. To our knowledge, this is the first of its kind article discussing the reversal of fatty liver with LAI in patients presenting with acute pancreatitis.

\section{Conflict of Interest}

Nil.

\section{Bibliography}

1. Hamer Okka W., et al. "Fatty liver: imaging patterns and pitfalls”. Radiographics 26 (2006): 1637-1653.

2. Rehm Jürgen., et al. "Alcohol as a risk factor for liver cirrhosis: a systematic review and meta-analysis". Drug and Alcohol Review 29 (2010): 437-445.

3. Samokhvalovet Andriy V., et al. "Alcohol consumption as a risk factor for acute and chronic pancreatitis: a systematic review and a series of meta-analyses". EBio Medicine 12 (2015): 19962002.

4. Singhvi Ajay., et al. "Coexistence of alcohol-related pancreatitis and alcohol-related liver disease: A systematic review and meta-analysis". Pancreatology 20 (2020): 1069-1077.

5. Rusyn Ivan and Ramon Bataller. "Alcohol and toxicity". Journal of Hepatology 59 (2013): 387-388.

6. Clemens Dahn L and Katrina J Mahan. "Alcoholic pancreatitis: Lessons from the liver". World Journal of Gastroenterology 16 (2010): 1314.

7. Morgan Timothy R. "Treatment of alcoholic liver disease". Gastroenterology and Hepatology 13 (2017): 425.

8. Limanond Piyaporn., et al. "Macrovesicular hepatic steatosis in living related liver donors: correlation between CT and histologic findings". Radiology 230 (2004): 276-280.

9. Angulo Paul. "Nonalcoholic fatty liver disease". The New England Journal of Medicine 346 (2002): 1221-1231.

10. Bellentani S., et al. "Prevalence of and risk factors for hepatic steatosis in Northern Italy". Annals of Internal Medicine 132 (2000): 112-117. 
11. El-Hassan AY., et al. "Fatty infiltration of the liver: analysis of prevalence, radiological and clinical features and influence on patient management". The British Journal of Radiology 65 (1992): 774-778.

12. Kammen BF., et al. "Focal fatty infiltration of the liver: analysis of prevalence and CT findings in children and young adults". American Journal of Roentgenology 177 (2001): 1035-1039.

13. Shen Lei., et al. "Prevalence of nonalcoholic fatty liver among administrative officers in Shanghai: an epidemiological survey". World Journal of Gastroenterology 9 (2003): 1106-1110.

14. Luyckx FH., et al. "Liver abnormalities in severely obese subjects: effect of drastic weight loss after gastroplasty". International Journal of Obesity and Related Metabolic Disorders 22(1998): 222-226.

15. Nomura H., et al. "Prevalence of fatty liver in a general population of Okinawa, Japan". Japanese Journal of Medicine 27 (1988): 142-149.

16. Global status report on alcohol and health World Health Organization". Edited by Vladimir Poznyak and Dag Rekve (2018).

17. Rehm Jürgen., et al. "Alcohol as a risk factor for liver cirrhosis: a systematic review and meta-analysis". Drug and Alcohol Review 29 (2010): 437-445.

18. Samokhvalov Andriy V., et al. "Alcohol Consumption as a Risk Factor for Acute and Chronic Pancreatitis: A Systematic Review and a Series of Meta-analyses". EBio Medicine 2 (2015): 1996-2002.

19. Baptista Ameha., et al. "Alcoholic Liver Disease: Morphological Manifestations". The Lancet 317 (1981): 707-711.

20. Utne HE and K Winkler. "Hepatic and extrahepatic elimination of ethanol in cirrhosis. With estimates of intrahepatic shunts and Km for ethanol elimination". Scandinavian Journal of Gastroenterology 15 (1980): 297-304.

21. Seitz Helmut K., et al. "Alcoholic liver disease". Nature Reviews Disease Primers 4.1(2018): 16.

22. Mann Robert E., et al. "The epidemiology of alcoholic liver disease". Alcohol Research and Health 27 (2003): 209-219.

23. Osna Natalia A., et al. "Alcoholic Liver Disease: Pathogenesis and Current Management". Alcohol Research 38 (2017): 147161.
24. Vohra Sandeep., et al. "Preoperative CT evaluation of potential donors in living donor liver transplantation". Indian Journal of Radiology and Imaging 24 (2014): 350-359.

25. National Health Service Alcohol Related Liver disease (2018).

26. Tommolino, Emily. Fatty Liver (2018).

27. Jackson Whitney. "Alcohol-Related Liver Disease" (2021).

\section{Volume 4 Issue 8 August 2021 \\ (C) All rights are reserved by Venkatesh Kasi Arunachalam., et al.}

